\title{
CpG hypermethylation of cellular retinol-binding protein 1 contributes to cell proliferation and migration in bladder cancer
}

\author{
KAZUKI TOKI ${ }^{1}$, HIDEKI ENOKIDA ${ }^{1}$, KAZUMORI KAWAKAMI ${ }^{1}$, TAKESHI CHIYOMARU ${ }^{1}$, \\ SHUICHI TATARANO ${ }^{1}$, HIROFUMI YOSHINO ${ }^{1}$, YOUSUKE UCHIDA ${ }^{1}$, KAZUYA KAWAHARA ${ }^{2}$, \\ KENRYU NISHIYAMA ${ }^{1}$, NAOHIKO SEKI ${ }^{3}$ and MASAYUKI NAKAGAWA ${ }^{1}$ \\ ${ }^{1}$ Department of Urology, Graduate School of Medical and Dental Sciences, Kagoshima University; \\ ${ }^{2}$ Kawahara Nephro-urology Clinic, Kagoshima; ${ }^{3}$ Department of Functional Genomics, \\ Graduate School of Medicine, Chiba University, Chiba, Japan
}

Received July 27, 2010; Accepted September 24, 2010

DOI: 10.3892/ijo_00000789

\begin{abstract}
We have previously reported a simple technique that combines microarray data from clinical bladder cancer (BC) specimens with those from a BC cell line (BOY) treated with a pharmacological demethylating agent [5-aza-2'-deoxycytidine $(5-a z a-d C)]$ to find candidate genes that have tumor suppressive functions. We focused on the cellular retinolbinding protein 1 (CRBPI) gene that was selected by using the microarray data. As CRBP1 regulates intracellular retinoic acid (vitamin $\mathrm{A}$ ) homeostasis, which is involved in morphogenesis, and cellular proliferation and differentiation, the loss of CRBP1 could cause tumorigenesis in BC. We hypothesized that the inactivation of the CRBPl gene through $\mathrm{CpG}$ methylation contributes to cell viability, including the migration and invasion activity of human BC cells. After the 5-aza-dC treatment, the mRNA and protein expression levels of CRBP1 markedly increased in all BOY and T24 BC cell lines. Combined bisulfite-restriction analysis and bisulfite DNA sequencing revealed that promoter CpG hypermethylation existed in 28 out of the $65 \mathrm{BCs}(43 \%)$ and in none of the 16 normal bladder epithelia (NBEs). Conversely, CRBP1 mRNA expression in the BCs was significantly lower than that in the NBEs $(0.63 \pm 0.11$ vs. $4.92 \pm 0.80, p<0.0001)$. We found significant inhibition of cell growth $(\mathrm{p}<0.0001)$ and migration $(\mathrm{p}<0.0001)$ in the CRBP1 stable transfectants compared to the control cell line, in a cell proliferation and wound-healing assay, respectively. In conclusion, the aberrant $\mathrm{CpG}$ hypermethylation of the $C R B P 1$ gene promoter could be involved in the development of $\mathrm{BC}$. We demonstrate here
\end{abstract}

Correspondence to: Dr Hideki Enokida, Department of Urology, Graduate School of Medical and Dental Sciences, Kagoshima University, 8-35-1 Sakuragaoka, Kagoshima 890-8520, Japan E-mail: enokida@m.kufm.kagoshima-u.ac.jp

Key words: microarray, cellular retinol-binding protein 1, bladder cancer, methylation for the first time that the CRBPI gene could have a tumor suppressive function in $\mathrm{BC}$.

\section{Introduction}

Bladder cancer (BC) is among the five most common malignancies worldwide, and it is the second most common tumor of the genito-urinary tract and the second most common cause of death in patients with genito-urinary tract malignancies (1). Although $70-80 \%$ of BCs are initially classified as nonmuscle invasive, $\sim 70 \%$ of them recur. Of these recurring tumors, $10-15 \%$ proceed to muscle invasion and metastasis (2). Therefore, it is crucial to find what novel mechanisms are involved in $\mathrm{BC}$ invasion and metastasis.

As is often the case with other human malignancies, the transcriptional silencing of tumor suppressor genes through the methylation of $\mathrm{CpG}$ islands in promoter regions, is considered to be an important and early stage of carcinogenesis in human $\mathrm{BC}$ (3). We previously developed a simple technique that combines microarray data from clinical $\mathrm{BC}$ specimens with those from a BC cell line (BOY) treated with a pharmacological demethylating agent [5-aza-2'-deoxycytidine (5-aza-dC)] (4-6). In these previous studies, we identified 20 genes that were considered to be methylated in human BC (shown here in Table III). Based on that profile, we demonstrated that the COL1A2 and FHL1 genes have a tumor suppressive function and that they were frequently inactivated in $\mathrm{BC}$ due to the $\mathrm{CpG}$ hypermethylation of the promoter region $(5,6)$. Thus, we believe that our method is quick and efficient in finding the methylated gene profile that is involved in cancer development. In the present study, we focused on the cellular retinol-binding protein 1 (CRBPl), which was among the candidate genes.

Retinol (vitamin A) is a fat-soluble micronutrient and occurs in nature in various forms (retinoids). In vivo, it functions as a form of retinoic acid (RA), which has a close relationship with important cellular functions such as morphogenesis, and cellular proliferation and differentiation (7). Retinoids are bound to CRBPs (types 1,2 and 3) in the cytoplasm. CRBP1 is widely distributed throughout the body, whereas CRBP2 and 3 demonstrate tissue-specific expression 
(8). Of these three, CRBP1 is thought to be essential for vitamin A homeostasis. The importance of this gene product in vivo was documented in CRBP1 knockout mice, which demonstrated squamous keratinizing metaplasia (9). The loss of CRBP1 expression due to the $\mathrm{CpG}$ hypermethylation of its promoter region has been found in various human cancer cell lines (10). Studies have also demonstrated that the $\mathrm{CpG}$ hypermethylation of the gene promoter is frequently observed in various cancers such as lymphoma, gallbladder and renal cell carcinoma, as well as breast, esophageal, gastric and prostate cancers (11-17). In terms of BC, certain studies have reported the promoter hypermethylation of CRBP1. However, they have validated neither the expression levels of CRBP1 transcripts nor its functional role in BC development $(11,18)$. Another study has also demonstrated that the mRNA expression levels of $C R B P 1$ were up-regulated in cervical cancer (19). Thus, the functional role of $C R B P 1$ has not been thoroughly investigated in $\mathrm{BC}$ and requires further study.

In the present study, we hypothesized that $C R B P 1$ was densely methylated in its promoter region and had a tumor suppressive function in BC. In order to test this, we examined the methylation status and mRNA expression in $\mathrm{BC}$ cell lines and clinical BC specimens by using combined bisulfiterestriction analysis (COBRA) and real-time RT-PCR. In addition, we established stable transfectants of $C R B P 1$ for gain-of-function studies and compared their proliferation, migration, and invasion activities with those of the control $\mathrm{BC}$ cell lines.

\section{Materials and methods}

Clinical samples. The tissue specimens were from $122 \mathrm{BC}$ patients who underwent transurethral resection (TUR) or radical cystectomy at Kagoshima University Hospital between 2003 and 2008. We also used 25 pathologicallyproven normal bladder epithelium (NBE) samples derived from organ-confined prostate cancer patients who underwent prostatectomy. The patient backgrounds and clinicopathological characteristics are summarized in Table I. Each tumor was staged and graded according to the TNM staging system (20), the Japanese Urological Association and the Japanese Society of Pathology (21). Our study was approved by the Bioethics Committee of Kagoshima University and prior written informed consent and approval were given by all patients.

Cell culture and 5-aza-dC treatment. We used two human $\mathrm{BC}$ cell lines. BOY was established in our laboratory from an Asian male 66-year-old patient who was diagnosed with stage III bladder cancer with lung metastasis (22) and T24 was obtained from the American Type Culture Collection. These cell lines were maintained in minimum essential medium which was supplemented with $10 \%$ fetal bovine serum, $1 \%$ L-glutamine and $1 \%$ penicillin/streptomycin in a humidified atmosphere of $5 \% \mathrm{CO}_{2}$ and $95 \%$ air at $37^{\circ} \mathrm{C}$. In order to screen for epigenetic alterations of gene methylation, these cells were treated with $1 \mu \mathrm{M}$ of the DNA methyltransferase inhibitor, 5-aza-dC (Sigma-Aldrich, St. Louis, MO, USA). Cultured cells were harvested after seven days of exposure to 5-aza-dC, and the genomic DNA and total RNA were extracted.
Table I. Patient characteristics.

\begin{tabular}{|c|c|}
\hline \multicolumn{2}{|l|}{$\mathrm{BC}$} \\
\hline Total number & 122 \\
\hline Median age, years (range) & $75(33-100)$ \\
\hline \multicolumn{2}{|l|}{ Gender } \\
\hline Male & 80 \\
\hline Female & 42 \\
\hline \multicolumn{2}{|l|}{ Stage } \\
\hline Tis & 1 \\
\hline Superficial (pTa) & 21 \\
\hline Invasive ( $\geq \mathrm{pT} 1)$ & 100 \\
\hline \multicolumn{2}{|l|}{ Grade } \\
\hline G1 & 11 \\
\hline G2 & 72 \\
\hline G3 & 39 \\
\hline \multicolumn{2}{|l|}{ Operation } \\
\hline Cystectomy & 16 \\
\hline TUR-Bt & 106 \\
\hline \multicolumn{2}{|l|}{ Recurrence } \\
\hline Recurrence (+) & 61 \\
\hline Recurrence (-) & 50 \\
\hline Unknown & 11 \\
\hline Follow-up period, days (range) & $513(13-1440)$ \\
\hline \multicolumn{2}{|l|}{ NBE } \\
\hline Total number & 25 \\
\hline Median age, years (range) & $66(58-76)$ \\
\hline \multicolumn{2}{|l|}{ Gender } \\
\hline Male & 19 \\
\hline Female & 6 \\
\hline
\end{tabular}

Nucleic acid extraction. Genomic DNA from the BCs and NBEs was extracted with QIAamp tissue kits (Qiagen, Valencia, CA) after the microdissection of $10-\mu$ m-thick paraffin-embedded sections and this was precipitated with ethanol. Total RNA was isolated from the frozen fresh tissues and BOY cells with Isogen (Nippon Gene, Tokyo, Japan) following the manufacturer's instructions. The concentrations of DNA and RNA were determined spectrophotometrically and their integrity was verified by gel electrophoresis. The RNA quality was checked in a BioPhotometer ${ }^{\mathrm{TM}}$ (Eppendorf, Tokyo, Japan).

cDNA preparation and conventional RT-PCR. We synthesized first strand cDNA with $1 \mu \mathrm{g}$ of total RNA using random primers and TaqMan reverse transcription reagents (High Capacity cDNA Reverse Transcription Kit; Applied Biosystems, Foster City, CA, USA). For conventional RTPCR, glyceraldehyde-3-phosphate 8 dehydrogenase (GAPDH) was used as the internal control. The primer sequences and RT-PCR conditions are listed in Table II. The PCR products were electrophoresed on $2 \%$ agarose gels.

Real-time quantitative RT-PCR. Gene specific PCR products were continuously measured with an Applied Biosystems 7300 Fast Real-Time PCR System following the instructions of the manufacturer. The initial PCR step was a 10-min hold at $95^{\circ} \mathrm{C}$ and the 45 cycles consisted of a 15 -sec denaturation step at $95^{\circ} \mathrm{C}$ followed by 1 -min annealing/extension at $60^{\circ} \mathrm{C}$. 
Table II. Primer sequences and PCR conditions.

Primer sequence $\left(5^{\prime}-3^{\prime}\right)$

Product size (bp)

Annealing temperature, time (cycle)

\section{Conventional RT-PCR}

CRBP1-S (sense)

CRBP1-AS (antisense)

GAPDH-S (sense)

GAPDH-AS (antisense)

COBRA

PAN-S1 (sense)

GTGGTTGTTGAGTGTGAGAAG

442

PAN-AS1 (antisense)

PAN-S2 (sense)

CATCTTCCAATACCCAATAAAATC

PAN-AS2 (antisense)
GTTGAGTGTGAGAAGTTAATGG

TTGTGGCCAAACTGGCTCCA

GAAGGTGAAGGTCGGAGTCA

CACTTGATTTTGGAGGGATCTC

CTAAAACCAATTAACCACAAAC $55^{\circ} \mathrm{C}, 30 \sec (35)$

$55^{\circ} \mathrm{C}, 30 \sec (35)$

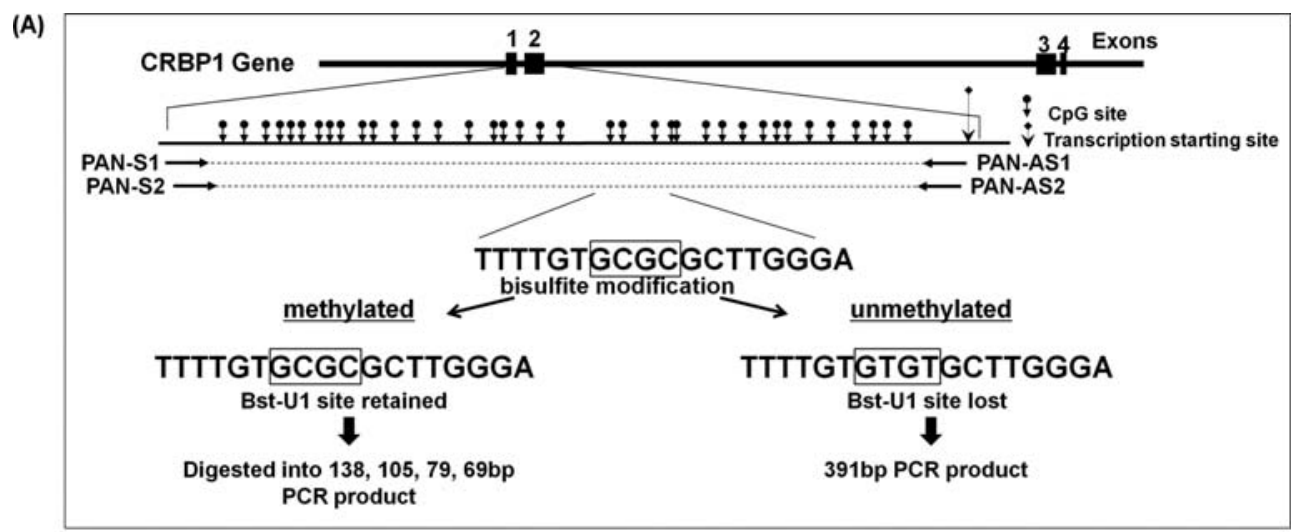

(B)
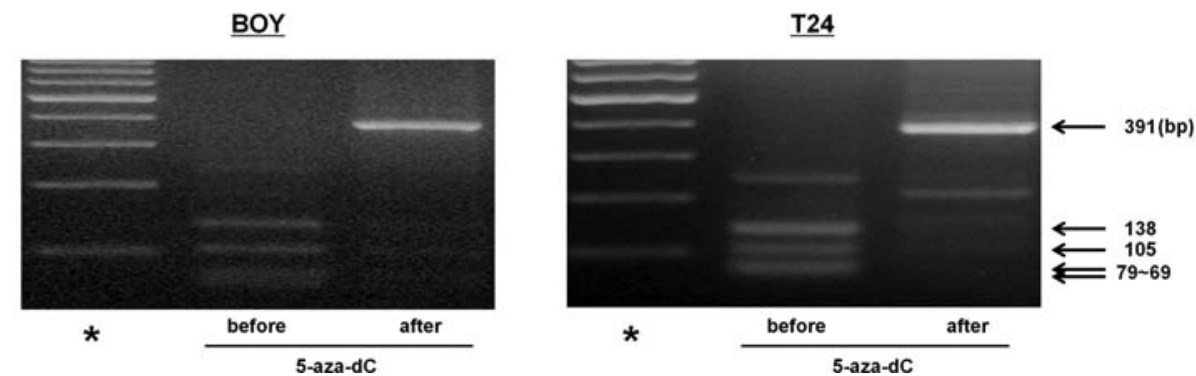

Figure 1. (A) Schematic representation of location of $\mathrm{CpG}$ sites and primers designed within the functional promoter of the $C R B P 1$ gene. Vertical arrows indicate $\mathrm{CpG}$ sites. Horizontal arrows indicate the positions of primers used. First, universal PCR was performed with the universal primers, PAN-S and PAN-AS, which do not carry any CpG sites. The original sequence for the target of COBRA is shown in the upper middle section. After bisulfite modification, the sequence changed into methylated (lower-left) and unmethylated patterns (lower-right). (B) Analysis of CRBP1 promoter methylation by COBRA. BstUI restriction enzyme digestion was performed to distinguish methylated and unmethylated alleles. The restriction site (CGCG) is recognized and digested by Bst $\mathrm{UI}$ when the site is methylated. Both the control BOY and T24 showed four fragments $(138,105,79$ and $69 \mathrm{bp})$, indicating that these were completely methylated. After treatment with 5-aza-dC, a 391-bp fragment appeared along with digested fragments, indicating that these were partially methylated. *100-bp DNA ladder.

All reactions were performed in triplicate and a negative control lacking cDNA was included. TaqMan ${ }^{\circledR}$ probes, primers for CRBP1 (P/N: Hs00161252_m1, Applied Biosystems) and human $18 S$ ribosomal RNA ( $r R N A$ ) (Applied Biosystems), were assay-on-demand gene expression products. The expression of CRBPI mRNA was normalized to the amount of human $18 S$ rRNA in the same cDNA using the standard curve method provided by the manufacturer.
DNA methylation analysis. In order to screen larger numbers of samples for CRBP1 promoter demethylation, we used COBRA, which produces restriction fragments from bisulfitetreated DNA only if methylation is present (Fig. 1A). A total of 65 BCs and 16 NBEs were only available for the study, as certain samples had insufficient amounts of tissue for the extraction of enough DNA for analysis. Genomic DNA (100 ng) was subjected to sodium bisulfite modification 
Table III. Top 20 methylated genes in BC.

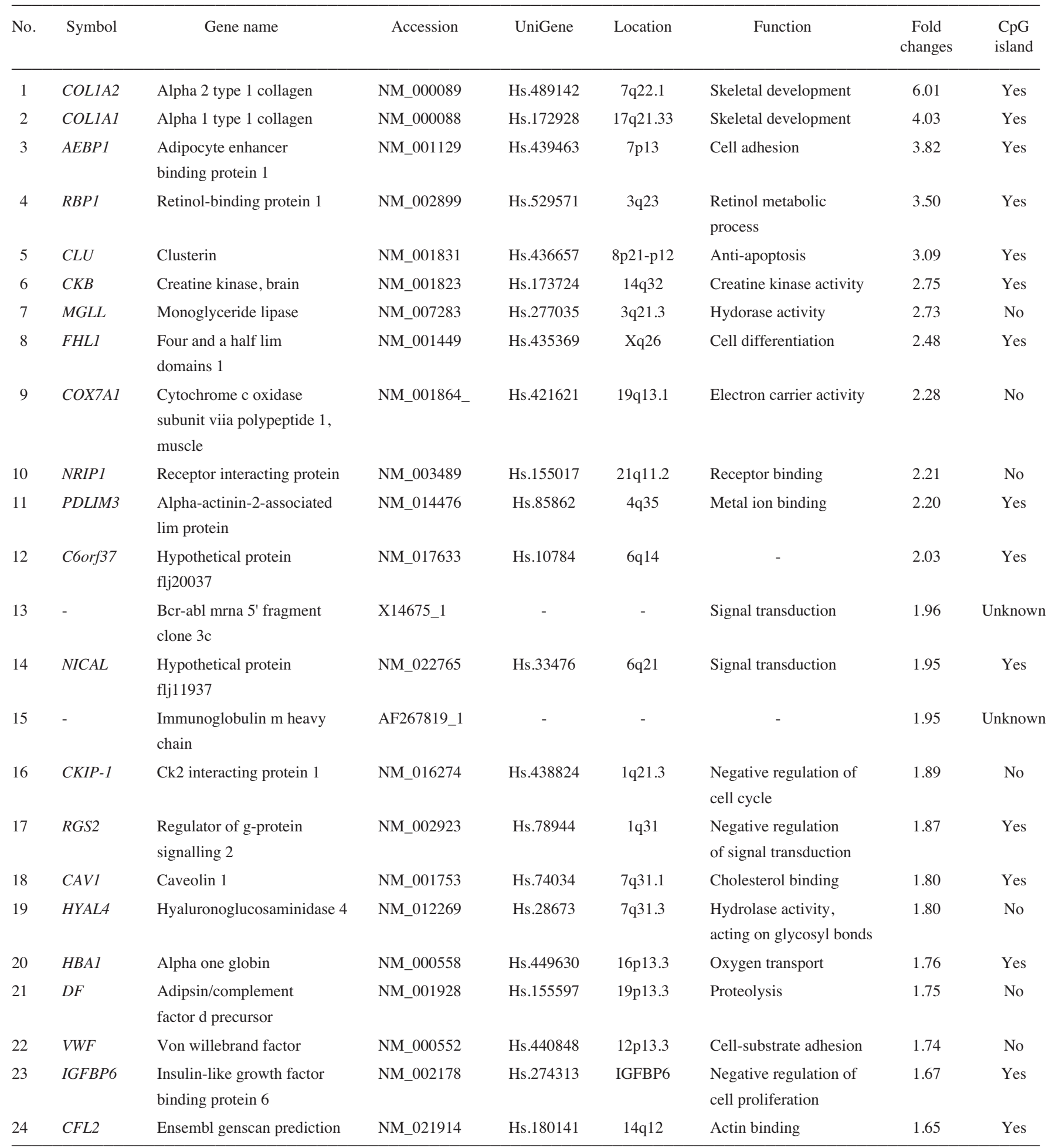

using the EpiTect Bisulfite Kit (Qiagen). DNA modified with sodium bisulfite was selectively amplified by first-round PCR with the primers Pan-sense 1 (PAN-S1) and Pan-antisense 2 (PAN-AS1), followed by second-round PCR with the primers (PAN-S2 and PAN-AS2), of which neither primer set carried any $\mathrm{CpG}$ sites (Fig. 1A). The primer sequences and PCR conditions are summarized in Table II. For COBRA, an equal amount of sodium bisulfite PCR product was digested with Bst UI (New England Biolabs, Hitchin, UK) at $60^{\circ} \mathrm{C}$ for
$2 \mathrm{~h}$. Digested fragments were visualized by the ethidium bromide staining of DNA, and were electrophoresed through a 2\% agarose gel. Under these PCR conditions, we obtained DNA fragments of $391 \mathrm{bp}$, which were cleaved into fragments of $138,105,79$ and 69 bp by digestion with Bst $\mathrm{U} 1$ when CpG was methylated. CpGenome ${ }^{\mathrm{TM}}$ Universal Methylated DNA and CpGenome ${ }^{\mathrm{TM}}$ Universal Unmethylated DNA Sets (Millipore, Billerica, MA, USA) were used as the positive and negative controls for the methylated alleles, respectively. 
(A)

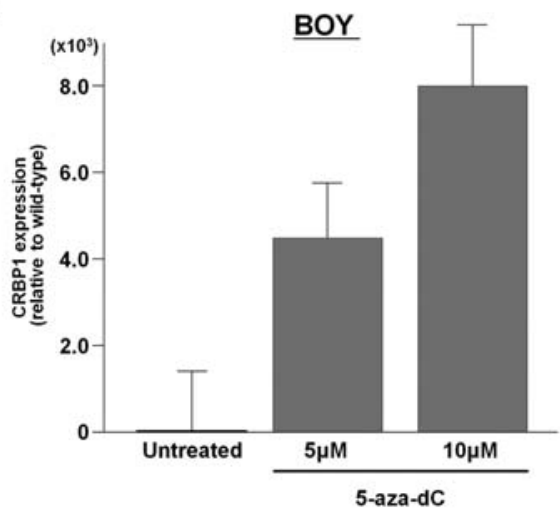

(B)

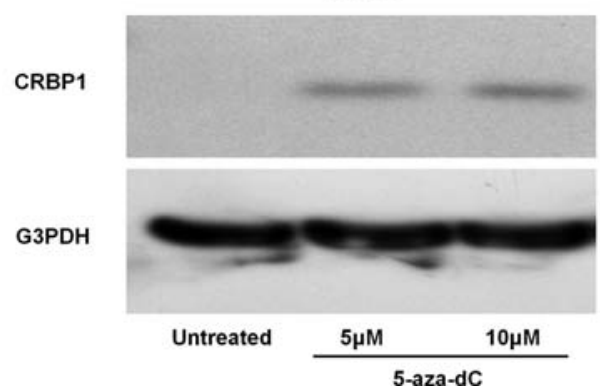

$\underline{T 24}$

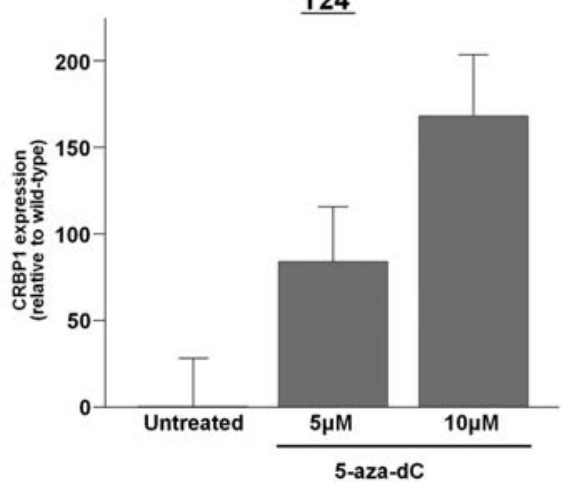

$\underline{T 24}$

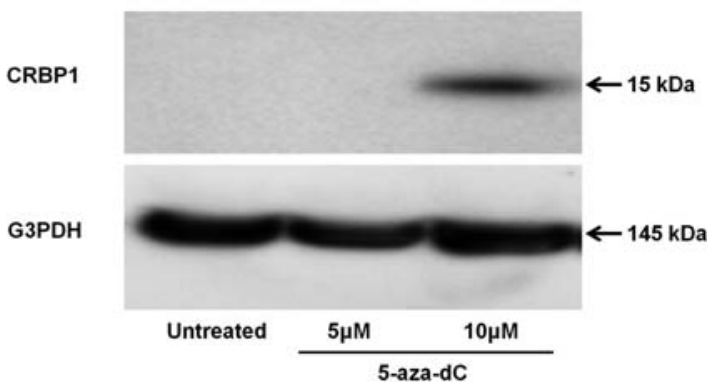

Figure 2. CRBP1 expression in BC cells before and after treatment with 5-aza-dC. (A) CRBP1 mRNA expressions were markedly increased in both cell lines after 5-aza-dC treatment in a dose-dependent manner. (B) CRBP1 protein bands (15 kDa) were restored in both cell lines after 5-aza-dC treatment.

Bisulfite DNA sequencing analysis. Bisulfite-modified DNA was amplified using a pair of universal primers, PAN-S1 and PAN-AS1, followed by a second nested PCR with the primers, PAN-S2 and PAN-AS2, in a total volume of $20 \mu 1$. The PCR conditions are shown in Table II. Direct bisulfite DNA sequencing of the PCR products using a forward PAN-S2 primer was carried out following the manufacturer's instructions (Applied Biosystems).

Immunoblotting. Total protein lysate was prepared with a detergent lysis buffer in the presence of a protease inhibitor. The $20 \mu \mathrm{g}$ of protein lysate were separated by using NuPAGE on a $4-12 \%$ Bis-Tris gel (Invitrogen, Tokyo) and transferred into a PVDF membrane. Immunoblotting was done with a diluted (1:200) polyclonal CRBPI antibody (sc-8786, Santa Cruz Biotechnology, Santa Cruz, CA). After being washed, the membrane was incubated with donkey anti-goat IgG horseradish peroxidase conjugate (Bio-Rad, Hercules, CA). Specific complexes were visualized with an echochemiluminescence detection system (GE Healthcare, Fairfield, CT, USA).

Construction of CRBPI expression vectors and transfection to $B C$ cell lines. The $C R B P I$ vector was constructed by inserting full-length $C R B P I \mathrm{cDNA}$ into the $\mathrm{BamHI}$ and $\mathrm{XbaI}$ restriction sites of the pBApo-CMV NeoTM vector (Takara Bio, Otsu, Japan). The CRBPl- and the non-targeting (control) vectors were transfected into the BOY and T24 cells by calcium phosphate co-precipitation. Both cells were split and grown in a selective medium with $1000 \mathrm{mg} / \mathrm{l}$ of G418. After two weeks, G418-resistant colonies were chosen and expanded in a medium containing $100 \mathrm{mg} / \mathrm{l}$ of G418.
XTT assays. For quantification of the cell proliferation of the CRBP1 transfectants, the controls and the wild-type BOY and T2 4 cells, the absorbance was measured at $492 \mathrm{~nm}$ after the cells had been exposed to XTT, which was metabolized by mitochondrial dehydrogenase to yield a formazan dye. In brief, the cells were transferred to 96 -well plates at $3 \times 10^{3}$ cells/well and incubated for $72 \mathrm{~h}$. Then, a $50 \mu \mathrm{l}$ solution of XTT $(0.3 \mathrm{mg} / \mathrm{ml})$ that contained $1.25 \mu \mathrm{M}$ of PMS was added to each well and the absorbance was measured after a further $4 \mathrm{~h}$ of incubation at $37^{\circ} \mathrm{C}$.

Wound-healing assay. The CRBP1 transfectants, controls and the wild-type BOY and T24 cells, were used in the 'wound-healing' assay to test the alteration of cell motility and migration. Cells were initially seeded uniformly into 60-mm culture plates with an artificial 'wound' carefully created at $0 \mathrm{~h}$. A P-20 pipette tip was used to scratch the subconfluent cell monolayer. Microphotographs were taken at 0,6 and $12 \mathrm{~h}$. Quantitative analysis of the percentage of wound healing was calculated using the distance across the wound $(\mathrm{n}=20)$ at 0,6 and $12 \mathrm{~h}$, divided by the distance measured at $0 \mathrm{~h}$ for each cell line.

Statistical analysis and annotation of gene function. The relationship between two groups and the numerical value obtained by real-time RT-PCR was analyzed using the MannWhitney $U$ test. The relationships between three groups and the numerical value were analyzed using the Bonferroniadjusted Mann-Whitney U test. The analysis software used was Expert StatView (version 4, SAS Institute Inc., Cary, NC, USA). The non-adjusted statistical level of significance of $\mathrm{p}<0.05$, corresponded to the Bonferroni-adjusted level of 
(A)

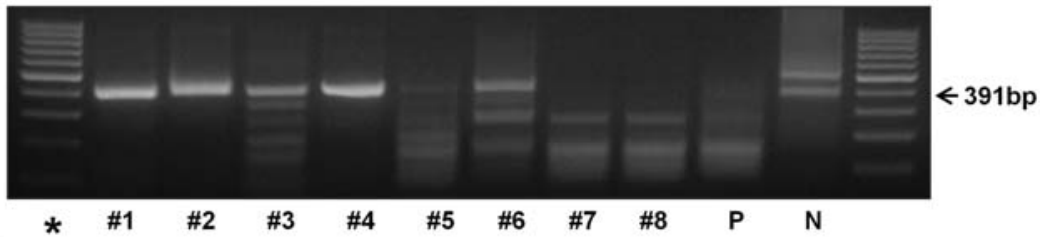

(B) Normal bladder epithelium (completely unmethylated)

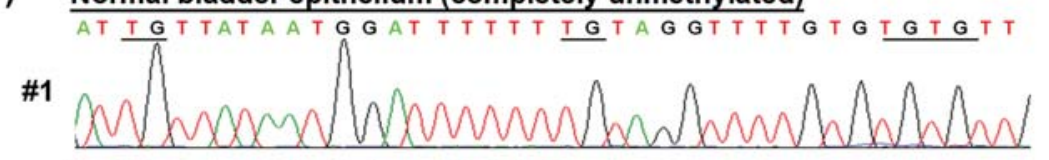

Bladder cancer (partially methylated)

AT CGTTATAATGGATTTTTTCGTAGGTTTT GTG CGCGTT

\#5

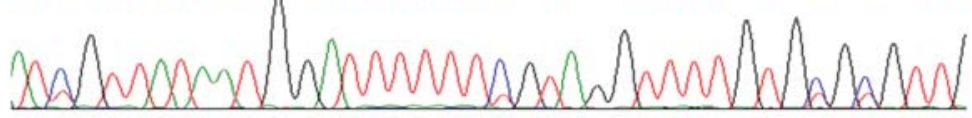

Bladder cancer (completely methylated)

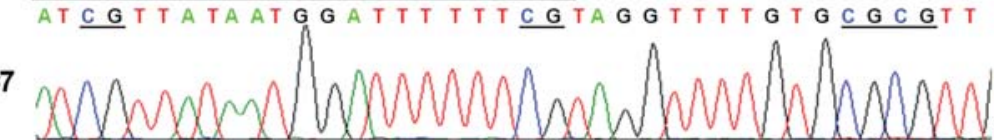

(C)

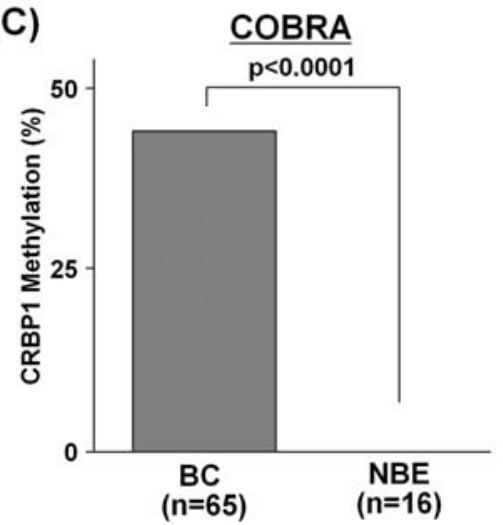

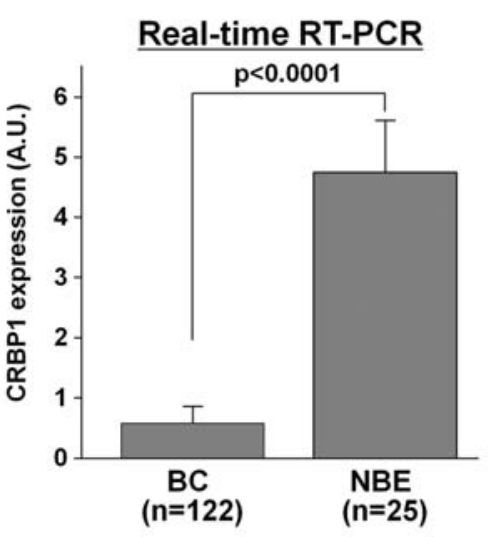

Figure 3. Methylation status of CRBP1 promoter region in clinical specimens. (A) COBRA classified the specimens into 'unmethylated' (lanes 1,2 and 4), 'partially methylated' (lanes 3,5 and 6), and 'completely methylated' (lanes 7 and 8). Universally methylated DNA was respectively used as the positive control (lane P) and unmethylated DNA was used as the negative control (lane N). "100-bp DNA ladder. (B) Bisulfite DNA sequencing confirmed 'unmethylated' (\#1), 'partially methylated' (\#5), and 'completely methylated' (\#7). Bars under the sequence indicate CpG sites. The results of the bisulfate DNA sequencing were consistent with those obtained by COBRA. (C) Methylation status and mRNA expression of CRBP1 in clinical specimens. (C, left) The promoter hypermethylation of the CRBP1 gene was detected in $43 \%$ of the BCs, whereas it was not detected in any of the NBEs. (C, right) CRBP1 mRNA expression in the BCs was significantly lower than that in the NBEs. All experiments were carried out in triplicate.

$\mathrm{p}<0.0167$ in the comparisons test comparing the three groups. The molecular function of the up- and down-regulated genes was classified into 17 categories as referenced in the Gene Ontology Consortium (http://www.geneontology.org/ index.shtml), i.e., apoptosis, anatomical structure development, cell cycle, cell differentiation and proliferation, gene expression, metabolic process, response to stimulus, regulation of gene expression, transport, regulation of apoptosis, cell proliferation, signal transduction and transcription, signal transduction, transport, transcription and others.

\section{Results}

Methylation status and altered CRBP1 expression in 5-aza-dCtreated $B C$ cell lines. Fig. 1 shows the methylation status of BOY and T24 analyzed with the COBRA assay. As the PCR product contains three 'CGCG' motifs, which are targeted by the restriction enzyme (Bst $\mathrm{UI})$, there could be four fragments
$(138,105,79$ and $69 \mathrm{bp})$ if a sample has complete methylation in all $\mathrm{CpG}$ sites, whereas there could be a single band of $391 \mathrm{bp}$ if a sample has complete unmethylation in these sites. If the $\mathrm{CpG}$ sites are partially methylated, the fragments could be observed in various combinations of these sizes. Before 5-aza-dC treatment, we found the four fragments (each of $138,105,79$ and $69 \mathrm{bp}$ ) and a fragment of $253 \mathrm{bp}$ (sum of 105, 79 and 69), but no 391-bp band was observed in either cell line. After $5 \mu \mathrm{M}$ of 5-aza-dC treatment, a 391-bp band appeared in BOY with various fragments of 148 bp (sum of 79 and 69) and $253 \mathrm{bp}$ (sum of 105, 79 and 69), while the band of 243 bp (sum of 138 and 105) in T24, indicated that complete demethylation was achieved by the demethylating agent even though methylated $\mathrm{CpG}$ sites partially remained (Fig. 1B). These cells were subjected to real-time RT-PCR to evaluate the mRNA expression levels of $C R B P 1$. The expression levels of $C R B P 1$ mRNA were markedly increased in a dose-dependent manner by 5 -aza-dC treatment (Fig. 2A). 
(A)
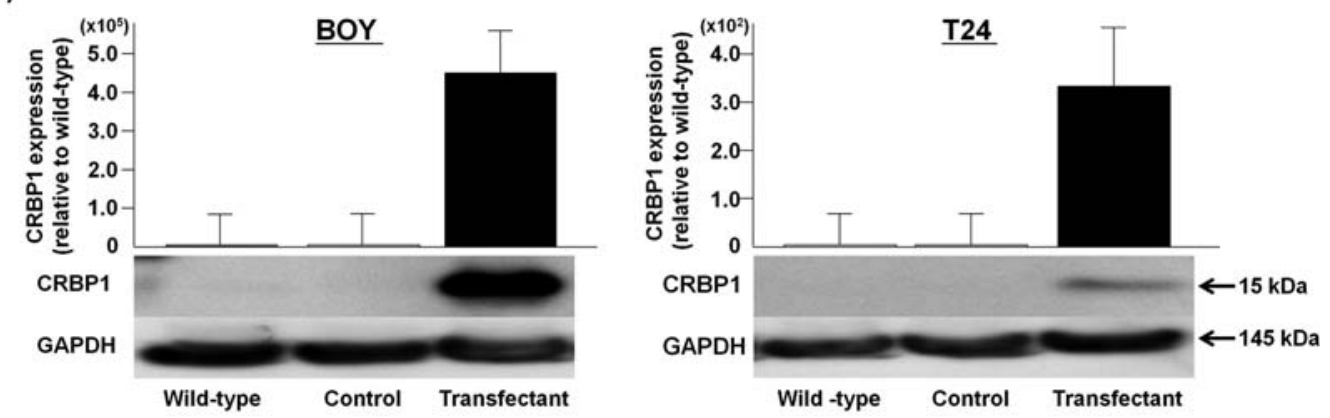

(B)
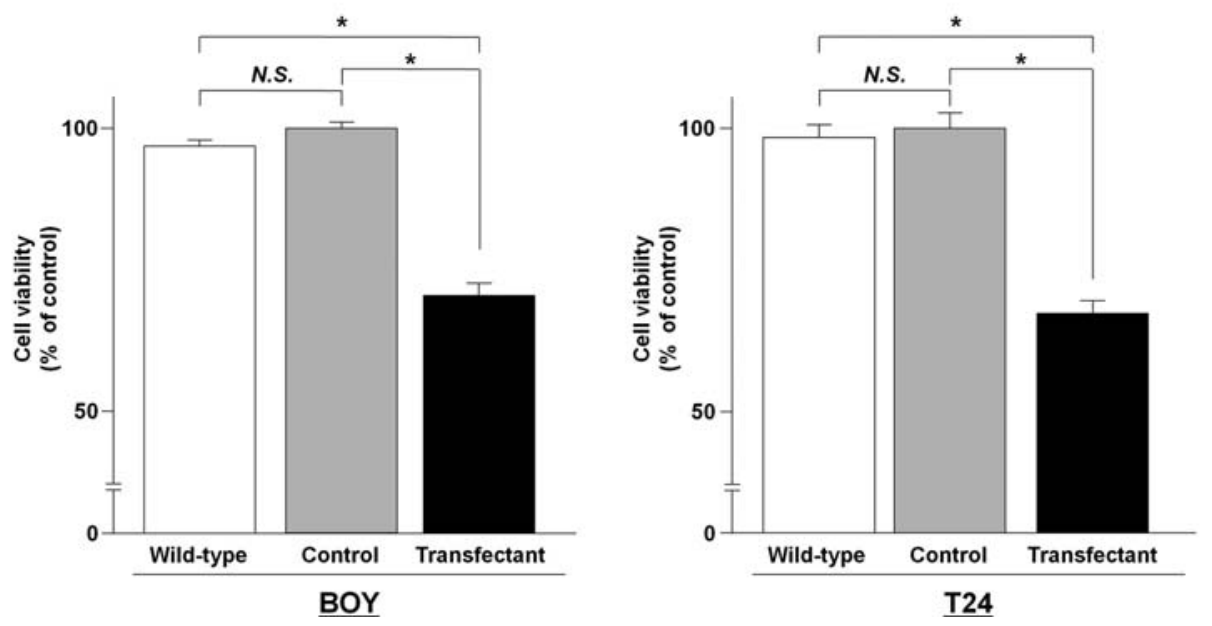

Figure 4. Effect of cell viabilities in CRBP1 transfectants by XTT assay. (A) CRBP1 expression determined by real-time RT-PCR and immunoblotting. (B) Cell viabilities determined by XTT assay. Cell viability inhibitions were observed in CRBP1-transfected BOY and T24 cell lines. "p $<0.0001$

Immunoblotting also demonstrated that the $15 \mathrm{kDa}$ CRBP1 protein was restored after 5 -aza-dC treatment in both cell lines (Fig. 2B).

DNA methylation status and mRNA expression of CRBP1 in clinical samples. Representative results of the COBRA assay in NBE (no. 1) and clinical BC (from nos. 2 to 8) samples are shown in Fig. 3A. A single band of 391 bp was observed in the sample nos. 1, 2 and 4, indicating that these $\mathrm{CpG}$ sites were completely unmethylated. The 391-bp band with various fragments was found in the sample nos. 3, 5 and 6 samples, indicating that these $\mathrm{CpG}$ sites were partially methylated. Sample nos. 7 and 8 had fragments without the 391-bp band, indicating these $\mathrm{CpG}$ sites were completely methylated. In Fig. 3B, we show the results of typical bisulfite DNA sequencing in the $\mathrm{BC}$ samples corresponding to COBRA. In sample no. 1 , there was a modest ' $\mathrm{T}$ ' and no ' $\mathrm{C}$ ' peak at any $\mathrm{CpG}$ site, substantiating that this was a completely unmethylated sample, as indicated by COBRA. Sample no. 5, which was diagnosed as partially methylated by COBRA, disclosed a strong ' $\mathrm{C}$ ' and modest ' $\mathrm{T}$ ' peak at each $\mathrm{CpG}$ site, corroborating that it was partially methylated. In sample no. 7, a strong ' $\mathrm{C}$ ' and no ' $\mathrm{T}$ ' peak at any $\mathrm{CpG}$ site substantiated that it was a completely methylated sample.

COBRA and bisulfite DNA sequencing revealed that there was promoter $\mathrm{CpG}$ hypermethylation in 28 out of the $65 \mathrm{BCs}(43 \%)$ and in none of the 16 NBEs (0\%) (Fig. 3C). Conversely, $C R B P 1$ mRNA expression in the $\mathrm{BCs}$ was significantly lower than that in the NBEs $(0.63 \pm 0.11$ vs. $4.92 \pm 0.80$, $\mathrm{p}<0.0001$ ) (Fig. 3C). We analyzed the relationship between the clinicopathological factors, but there was no correlation between the methylation status or mRNA expression of $C R B P 1$ and the patient characteristics (data not shown).

Identification of CRBP1 expression in transfected cells and effect of CRBP1 overexpression on cell growth. As we found very low expression levels of $C R B P 1 \mathrm{mRNA}$ in wild-type BOY and T24 cells, we established CRBP1 transfectants from the BOY and T24 cells. The expression levels of $C R B P 1$ mRNA in the BOY/CRBP1 transfectants were $\sim 450,000$-fold higher than those in the control or wild-type BOY cells, and the levels in the T24/CRBP1 transfectants were 400-fold higher than those in the control or wild-type T24 cells (Fig. 4A). Immunoblotting vigorously detected a $15-\mathrm{kDa}$ CRBP1 protein in the BOY/CRBP1 transfectants, and it was modestly detected in the T24/CRBP1 transfectants (Fig. 4A). In order to evaluate the relationship between $C R B P 1$ expression and cell growth, we performed XTT assays using the CRBP1 transfectants, control, and wild-type cells. Three independent XTT assays consistently demonstrated significant growth inhibitions in the CRBP1 transfectants compared to the control, and wild-type cells (\% of cell viability in BOY on day 3: $68.8 \pm 0.7,100 \pm 1.8$ and $95.7 \pm 0.8$, respectively, $\mathrm{p}<0.0001$, and that in $\mathrm{T} 24: 66.6 \pm 0.3$, $100 \pm 1.8$ and 99.5 \pm 0.8 , respectively, $\mathrm{p}<0.0001$ ) (Fig. 4B).

In order to examine cellular migration ability, we carried out wound-healing assays using the CRBP1 transfectants in comparison to their counterparts. Three independent wound- 
BOY

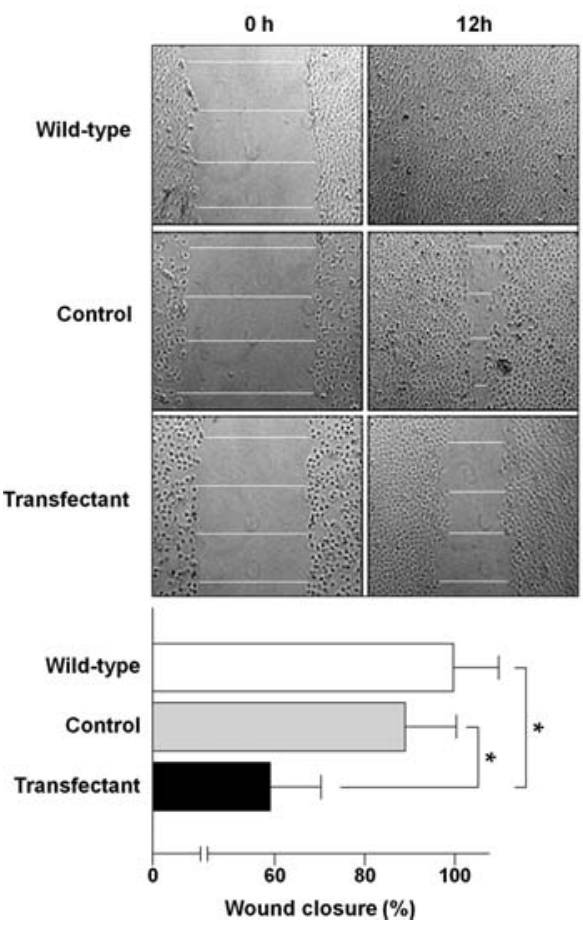

$\underline{T 24}$

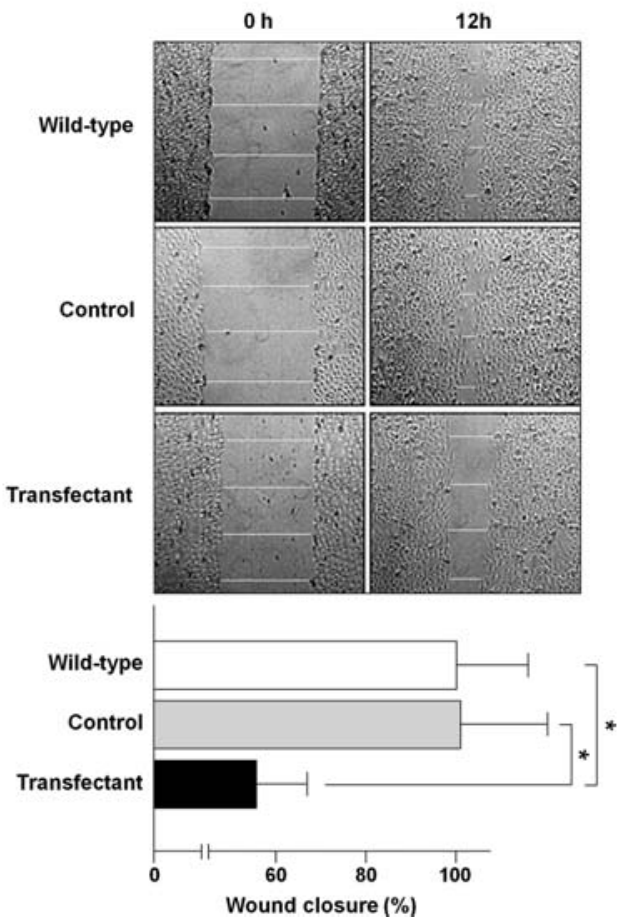

Figure 5. Percentage of wound closure corresponding to the distance between wound edges relative to the distance at time $0 \mathrm{~h}$ for each cell. Significant wound healing inhibition was observed in the CRBP1 transfectant after $12 \mathrm{~h}$ in comparison to the wild-type and the control. " $\mathrm{p}<0.0001$.

(A)

Up-regulated genes ( $n=1039)$

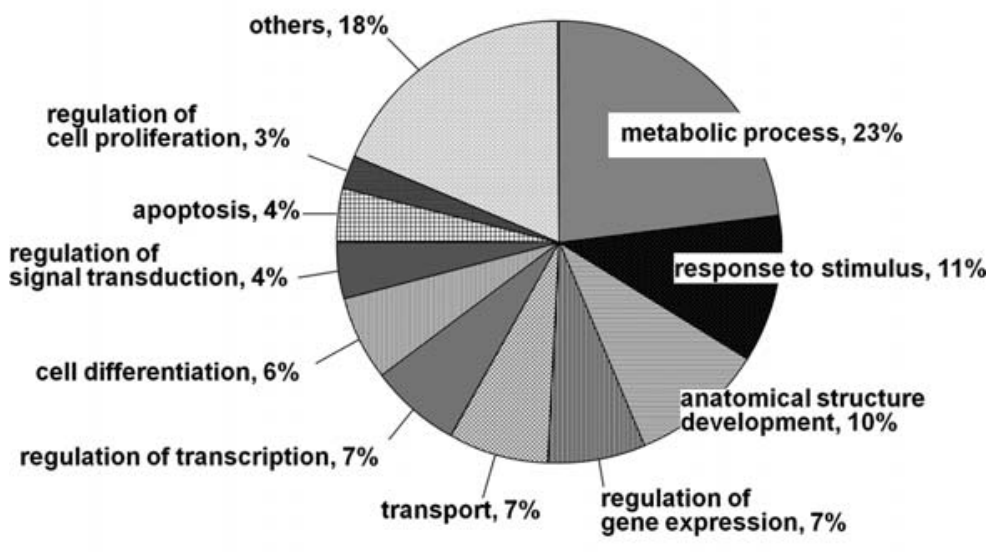

(B)

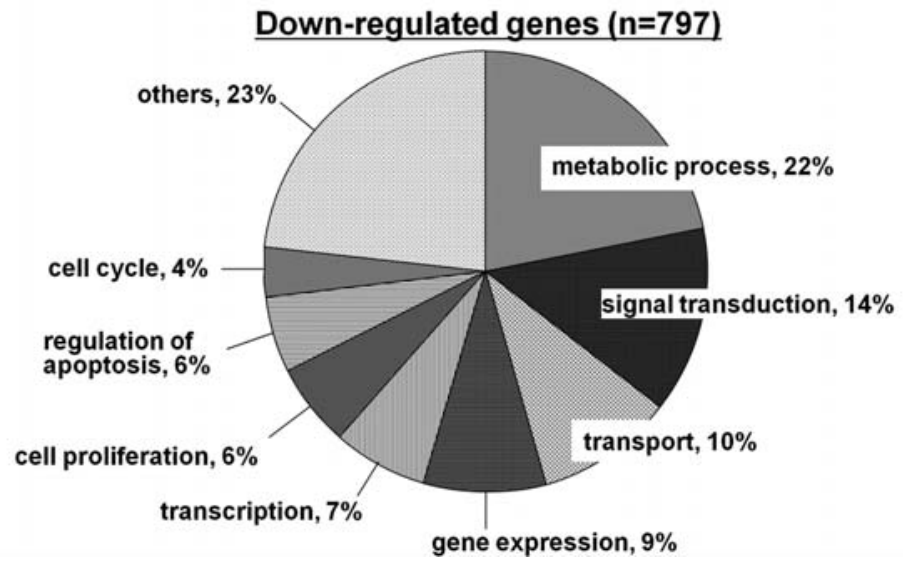

Figure 6. Distribution of altered expressions of functionally categorized genes in CRBP1 transfectants in comparison to the control transfectant. The functional features of the 1039 up- (A) and 797 down-regulated (B) genes were classified into 17 categories. 
healing assays demonstrated significant cell migration inhibitions in the CRBP1 transfectants after $48 \mathrm{~h}$ of treatment (\% of wound closure in BOY: $59.3 \pm 3.8,89.1 \pm 1.9$ and $100 \pm 10.8$, respectively, $\mathrm{p}<0.0001$, and that in $\mathrm{T} 24: 57 \pm 7.5$, $104.1 \pm 0.9$ and $100 \pm 0.7$, respectively, $\mathrm{p}<0.0001$ ) (Fig. 5).

Gene expression analysis for CRBPI overexpression. In order to gain further insight into which genes were affected by the activity of the $C R B P 1$ gene expression, we analyzed the gene expression profile of the $C R B P 1$ transfectants, in which the $C R B P 1$ protein was overwhelmingly expressed (Fig. 4A). We identified 1039 genes that were up-regulated $>2$-fold compared to the control transfectant (Fig. 6A). In contrast, 797 genes were down-regulated $<2$-fold (Fig. 6B). The functional annotations of the up- and down-regulated genes were classified into 17 categories (Fig. 6). Functionally, the up-regulated genes were involved in the metabolic process $(23 \%)$, response to stimulus $(11 \%)$, anatomical structure development $(10 \%)$, regulation of gene expression $(7 \%)$, transport $(7 \%)$, regulation of transcription $(7 \%)$, cell differentiation $(6 \%)$, regulation of signal transduction $(4 \%)$, apoptosis (4\%), regulation of cell proliferation (3\%) and others (18\%) (Fig. 6A). The down-regulated genes were involved in the metabolic process $(22 \%)$, signal transduction $(14 \%)$, transport $(10 \%)$, gene expression $(9 \%)$, transcription $(7 \%)$, cell proliferation $(6 \%)$, regulation of apoptosis $(6 \%)$, cell cycle (4\%) and others (23\%) (Fig. 6B).

\section{Discussion}

We have previously demonstrated that our profile for methylated genes in BC is preferable as we used microarray data from two simple hybridizations. In brief, we identified the genes methylated in human BC (Table III) by filtering the subset of genes restored after 5-aza-dC treatment in human $\mathrm{BC}$ cell lines from the down-regulated genes in the clinical BCs in our previous microarray (4). Based on this profile, we focused on the $C R B P 1$ gene in this study, and we confirmed that the promoter hypermethylation of $C R B P 1$ was significantly more frequent in BCs than in NBEs. Other sudies have also demonstrated that the promoter hypermethylation of $C R B P 1$ is a frequent event in various cancers, and that it inhibits the transcription of CRBP1 (10-18). However, there have not been many studies on the functional role of CRBP1 in BC. Consistent with previous studies on other malignancies, we also found $C R B P 1$ promoter hypermethylation and the frequent down-regulation of $C R B P 1 \mathrm{mRNA}$ in human BC. In addition, the demethylating agent restored $C R B P 1$ mRNA expression through the demethylation of the $C R B P 1$ promoter in $\mathrm{BC}$ cell lines, suggesting that the inactivation of the $C R B P 1$ gene by the promoter plays an important role in human BC development. The COBRA assays demonstrated that there were various methylation stages (complete and partial methylation as well as unmethylation) in the clinical BCs, suggesting that these degrees of methylation could contribute to a BC phenotype, which defines the malignant potential. These results led us to believe that it would be better to use a combination of RA and demethylating agents, such as aza-dC, for therapeutic strategies in patients with BC. There was no significant relationship in our cohort between the $C R B P 1$ expression and clinicopathological parameters including tumor stage and grade, and patient prognosis. However, a previous study demonstrated that CRBP1 gene expression was correlated with longer survival in laryngeal squamous cell carcinoma (23). The follow-up period was too short to evaluate prognosis in the present study.

It is known that $C R B P 1$ regulates intracellular RA transport, metabolism, and transcriptional activity (7). The loss of $C R B P 1$ could diminish RA transport and result in blockage of the transcription of RA-responsive genes that regulate cell proliferation. However, previous studies have supported the hypothesis that CRBP1 could be involved in the promotion of apoptosis through an increase in the RA metabolism in breast cancer cell lines $(17,24,25)$. As the suppression of apoptosis played a key role in BC carcinogenesis (26), the observed low expression of CRBP1 in our study could disrupt the RA-mediated apoptosis pathway in BC. In clinical terms, the functional role of the CRBPl gene in human cancers still remains controversial. Many studies have shown that it is down-regulated and functions as a tumor suppressor gene (11-18), whereas it is up-regulated in cervical cancer (19). In breast cancer, CRBP1 down-regulation promoted the progression of tumors through the inhibition of RA receptor activity (24). In terms of BC, RA treatment effectively reduced the recurrence rate and increased the survival time of superficial BC patients $(26,27)$. However, there have been no gain-of-function studies on the CRBPl gene. Therefore, we established CRBP1 transfectants using BC cell lines, and these significantly showed growth and migration inhibition in comparison to the controls. The data suggest that CRBP1 gene expression can cause a tumor suppressive function in BC through the intracellular RA metabolism. Further investigations are necessary to clarify whether these phenomena are critical in the development of BC.

In order to gain further insight into which genes are affected by $C R B P 1$ gene expression, we analyzed the gene expression profile of the CRBP1 transfectants. The functional annotations of the up-regulated genes after CRBP1 transfection were distributed among 17 categories including apoptosis, anatomical structure development, cell cycle, and cell differentiation and proliferation. The up-regulated gene categories in the CRBP1 transfectants include tumor suppressive categories, such as anatomical structure development, cell differentiation and apoptosis. However, the downregulated gene categories include oncogenic categories, such as signal transduction, transcription, cell proliferation and cell cycle. These results suggest that $C R B P 1$ activates other tumor suppressor genes and inactivates oncogenic genes in BC. Further investigations are required to test this hypothesis.

We concluded that the mechanism for CRBP1 downregulation in $\mathrm{BC}$ cells occurs through the $\mathrm{CpG}$ hypermethylation of the promoter region. To the best of our knowledge, we demonstrate for the first time that the CRBPI gene can have a tumor suppressive function in human $\mathrm{BC}$. The fact that the restored CRBP1 expression in the transfectants decreased cell growth and migration activity, indicates that this gene is a promising candidate for gene therapy of human BC. 


\section{Acknowledgements}

We thank Ms. Mutsumi Miyazaki for her excellent laboratory assistance.

\section{References}

1. Jemal A, Siegel R, Ward E, Hao Y, Xu J and Thun MJ: Cancer statistics, 2009. CA Cancer J Clin 59: 225-249, 2009.

2. Herr H, Konety B, Stein J, Sternberg CN and Wood DP Jr: Optimizing outcomes at every stage of bladder cancer: do we practice it? Urol Oncol 27: 72-74, 2009.

3. Enokida $\mathrm{H}$ and Nakagawa M: Epigenetics in bladder cancer. Int J Clin Oncol 13: 298-307, 2008.

4. Kawakami K, Enokida H, Tachiwada T, et al: Identification of differentially expressed genes in human bladder cancer through genome-wide gene expression profiling. Oncol Rep 16: 521-531, 2006.

5. Mori K, Enokida H, Kagara I, et al: $\mathrm{CpG}$ hypermethylation of collagen type I $\alpha 2$ contributes to proliferation and migration activity of human bladder cancer. Int J Oncol 34: 1593-1602, 2009.

6. Matsumoto M, Kawakami K, Enokida H, et al: $\mathrm{CpG}$ hypermethylation of human four-and-a-half LIM domains 1 contributes to migration and invasion activity of human bladder cancer. Int J Mol Med 26: 241-247, 2010.

7. Wolf G: Identification of a membrane receptor for retinolbinding protein functioning in the cellular uptake of retinol. Nutr Rev 65: 385-388, 2007.

8. Vogel S, Mendelsohn CL, Mertz JR, et al: Characterization of a new member of the fatty acid-binding protein family that binds all-trans-retinol. J Biol Chem 276: 1353-1360, 2001.

9. Ghyselinck NB, Båvik C, Sapin V, et al: Cellular retinolbinding protein I is essential for vitamin A homeostasis. EMBO J 18: 4903-4914, 1999.

10. Esteller M, Guo M, Moreno V, et al: Hypermethylationassociated inactivation of the cellular retinol-binding-protein 1 gene in human cancer. Cancer Res 62: 5902-5905, 2002.

11. Chu LC, Eberhart CG, Grossman SA and Herman JG: Epigenetic silencing of multiple genes in primary CNS lymphoma. Int J Cancer 119: 2487-2491, 2006.

12. Takahashi T, Shivapurkar N, Riquelme E, et al: Aberrant promoter hypermethylation of multiple genes in gallbladder carcinoma and chronic cholecystitis. Clin Cancer Res 10 6126-6133, 2004

13. Morris MR, Hesson LB, Wagner KJ, et al: Multigene methylation analysis of Wilms' tumour and adult renal cell carcinoma. Oncogene 22: 6794-6801, 2003.

14. Vasilatos SN, Broadwater G, Barry WT, et al: CpG island tumor suppressor promoter methylation in non-BRCAassociated early mammary carcinogenesis. Cancer Epidemiol Biomarkers Prev 18: 901-914, 2009.
15. Mizuiri H, Yoshida K, Toge T, et al: DNA methylation of genes linked to retinoid signaling in squamous cell carcinoma of the esophagus: DNA methylation of CRBP1 and TIG1 is associated with tumor stage. Cancer Sci 96: 571-577, 2005.

16. Shutoh M, Oue N, Aung PP, et al: DNA methylation of genes linked with retinoid signaling in gastric cancer expression of retinoid acid receptor-beta (RAR-beta), cellular retinol-binding protein 1 (CRBP1), and tazarotene-induced gene 1 (TIG1) was associated with DNA methylation. Cancer 104: 1609-1619, 2005.

17. Suzuki M, Shigematsu H, Shivapurkar N, et al: Methylation of apoptosis related genes in the pathogenesis and prognosis of prostate cancer. Cancer Lett 242: 222-230, 2006.

18. Brait M, Begum S, Carvalho AL, et al: Aberrant promoter methylation of multiple genes during pathogenesis of bladder cancer. Cancer Epidemiol Biomarkers Prev 17: 2786-2794, 2008.

19. Song JY, Lee JK, Lee NW, Jung HH, Kim SH and Lee KW: Microarray analysis of normal cervix, carcinoma in situ, and invasive cervical cancer: identification of candidate genes in pathogenesis of invasion in cervical cancer. Int J Gynecol Cancer 18: 1051-1059, 2008.

20. Sobin LH and Wittekind C: TNM Classification of malignant tumours. In: International Union Against Cancer (UICC). 6th edition. Wiley-Liss Publications, New York, pp199-202, 2002.

21. General Rules for Clinical and Pathological Studies on Bladder Cancer. In: The Japanese Urological Association, the Japanese Society of Pathology. 2nd edition. Kanehara Publishing Co., Tokyo, p80, 1993.

22. Ichimi T, Enokida H, Okuno Y, et al: Identification of novel microRNA targets based on microRNA signatures in bladder cancer. Int J Cancer 125: 345-352, 2009.

23. Peralta R, Baudis M, Vazquez G, et al: Increased expression of cellular retinol-binding protein 1 in laryngeal squamous cell carcinoma. J Cancer Res Clin Oncol 136: 931-938, 2010.

24. Kuppumbatti YS, Rexer B, Nakajo S, Nakaya K and Mira-yLopez R: CRBP suppresses breast cancer cell survival and anchorage-independent growth. Oncogene 20: 7413-7419, 2001.

25. Murphy TM, Perry AS and Lawler M: The emergence of DNA methylation as a key modulator of aberrant cell death in prostate cancer. Endocr Relat Cancer 15: 11-25, 2008.

26. Nishiyama H, Watanabe $J$ and Ogawa O: p53 and chemosensitivity in bladder cancer. Int J Clin Oncol 13: 282-286, 2008.

27. Hameed DA and el-Metwally TH: The effectiveness of retinoic acid treatment in bladder cancer: impact on recurrence, survival and TGF alpha and VEGF as end-point biomarkers. Cancer Biol Ther 7: 92-100, 2008. 